\title{
Emprendedores y proyectos fallidos de LEADER y PRODER en Andalucía en el periodo 2000-2006. Perfil y motivos de desistimiento
}

\section{Entrepreneurs and failed projects from LEADER and PRODER in Andalusia in the period 2000-2006. Profile and reasons for withdrawal}

\author{
Francisco Antonio Navarro Valverde ${ }^{1}$, Eugenio Cejudo García ${ }^{2}$ \\ y José Antonio Cañete Pérez ${ }^{3}$
}

RESUMEN

Durante 25 años el enfoque LEADER ha contribuido a incorporar nuevos principios a las recientes teorías del desarrollo neoendógeno. Para esta Iniciativa, se realiza un análisis de los proyectos que, habiendo sido inicialmente aprobados, no se llegaron a ejecutar para conocer el perfil del promotor, tipo de inversión y causas que provocaron su caída. Se han consultado los expedientes no ejecutados durante el periodo $2002-$ 2008 de los Grupos de Acción Local de la provincia de Granada y se han realizado entrevistas tanto a los técnicos como a los gerentes de tales Grupos. En estos proyectos se aprecia una prevalencia del emprendedor privado sobre el público y de los jóvenes y nuevos empresarios frente a los que no lo son. Las causas últimas de desistimiento van desde la inadecuada planificación y la falta de financiación, hasta las rigideces en tiempo y requisitos administrativos exigidos.

1 Departamento de Geografía Humana, Facultad de Filosofía y Letras, Campus Universitario de Cartuja, Universidad de Granada, Granada favalver@ugr.es

ORCID iD: http://orcid.org/0000-0001-5033-7603

2 Departamento de Geografía Humana, Facultad de Filosofía y Letras, Campus Universitario de Cartuja, Universidad de Granada, Granada cejudo@ugr.es

ORCID iD: http://orcid.org/0000-0003-2564-5887

3 Departamento de Geografía Humana, Facultad de Filosofía y Letras, Campus Universitario de Cartuja, Universidad de Granada, Granada joseaca@ugr.es

ORCID iD: http://orcid.org/0000-0003-1145-5900

Copyright: @ 2018 CSIC. Este es un artículo de acceso abierto distribuido bajo los términos de la licencia de uso y distribución Creative Commons Reconocimiento 4.0 Internacional (CC BY 4.0). 
Palabras Clave: enfoque LEADER; desarrollo rural neoendógeno; proyectos fallidos/ caídos; mujeres y jóvenes rurales; Andalucía.

\begin{abstract}
For 25 years, the LEADER approach has contributed to incorporate new principles to recent theories of neoendogenous rural development. For this Initiative, an analysis of the projects has been made that, having initially been approved, were not finished, in order to know the profile of the promoters, types of investment, and main causes of their failure. The files not executed during the period 2002-2008, from the Local Action Groups of the province of Granada, have been consulted, and exhaustive interviews, both with the technicians and the managers of such groups, they have been carried out. The projects analized show a prevalence of the private entrepreneur over the public, and the young and new entrepreneurs compared to those from other groups. Among the main final causes of failure are: inadequate planning and funding, not being able to provide the demanded documentation in time, and the rigidity, in terms of time and bureaucratic requirements, of such programmes.
\end{abstract}

KEY WORDS: LEADER approach; neoendogenous rural development; failed/fallen projects; rural women and youth; Andalusia.

Cómo citar este artículo/Citation: Navarro Valverde, Francisco Antonio, Cejudo García, Eugenio, Cañete Pérez, José Antonio (2018): "Emprendedores y proyectos fallidos de LEADER y PRODER en Andalucía en el periodo 2000-2006. Perfil y motivos de desistimiento", Estudios Geográficos, LXXIX/284, pp. 141-166

\title{
INTRODUCCIÓN
}

La iniciativa comunitaria de desarrollo rural LEADER (siglas francesas de Liaisons entre Activités de Developement de L'Economie Rural, y que significan en español Enlaces entre acciones para el desarrollo de la economía rural) ha aportado, en su aplicación en la Unión Europea (UE), significativos cambios en los territorios donde se ha implementado. Su origen está en la Reforma de los Fondos Estructurales de finales de la década de los 80 del siglo XX, dentro de las acciones «fuera de cuota» del FEDER en las que, con un porcentaje de gasto de en torno al 5\%, se ejecutaban iniciativas de ámbito comunitario, como respuesta a problemas comunes, a propuesta de la Comisión. Esta Reforma coincide en el tiempo con la aparición del documento El Futuro del Mundo Rural, de 1988. Como semilla para el desarrollo rural, esta Iniciativa incluye ocho principales principios o especificidades: diversificación económica, innovación, puesta en valor de los recursos endógenos, participación ascendente y toma de decisiones local; además de otros como son el carácter integrado, la cooperación y creación 
de redes entre territorios rurales, y la conformación de partenariados públicoprivados a través de Grupos de Acción Local (GAL).

Así, LEADER se ha ido aplicando a lo largo de 25 años, desde comienzos de la década de los 90 del siglo pasado. Desde LEADER I (1991-93), LEADER II (1995-99), LEADER + (2000-06), a las medidas específicas de LEADER en los respectivos Programas de Desarrollo Rural regionales aplicados con el Fondo Europeo Agrícola y de Desarrollo Rural (FEADER), 2007-2013 y en la actualidad, 2014-2020. Por su parte, en este trabajo también se aborda el Programa Operativo de Desarrollo Rural PRODER, programa afín entendido como un «LEADER a la española». En concreto, y para el periodo 2000-06, se analizan LEADER + y PRODER II.

El propósito de este trabajo es analizar los proyectos fallidos de esta Iniciativa, LEADER + y de PRODER, del periodo 2000-2006, aunque su tramitación se prolongó hasta 2008; para la región andaluza, por qué habiendo sido aprobados, y tras firmar el correspondiente contrato de ayuda con el correspondiente GAL, no se llegaron a ejecutar, las causas últimas que abocaron a que no se llegasen a realizar, al menos con financiación procedente de LEADER o PRODER; además de las tipologías de promotor y de inversión preponderantes. Todo ello contribuirá a conocer mejor el funcionamiento de la gestión de estos programas, además de buscar posibles errores en su implementación.

Existe una abundante bibliografía sobre la aplicación de LEADER como iniciativa y enfoque para el desarrollo endógeno en ámbitos rurales. Atendiendo a Barke y Newton (1997) se puede considerar la práctica más emblemática en desarrollo rural construida a partir de las teorías del desarrollo neo-endógeno, combinando y complementando los enfoques ascendente y descendente, los elementos endógenos y exógenos, la participación, las relaciones internasy externas, y los instrumentos de gobernanza y gobierno (Ray, 2006; Marsden, 2009). Puede ser considerado como un laboratorio para la experimentación en democracia participativa (Ray, 2000), y un nuevo modo de gobernanza para las áreas rurales (Goodwin, 1998; Marsden y Murdoch, 1998; Esparcia, Noguera y Pitarch, 2015). Para High y Nemes (2007) las instituciones para el desarrollo rural endógenas y exógenas se refuerzan más que oponerse unas a las otras. Recientemente, para Cheshire, Esparcia y Shucksmith (2015), y para Martínez, Sacristán y Yagüe (2015), entre los factores para explicar la resiliencia de los territorios rurales estarían las prácticas de desarrollo rural neo-endógeno, incluyendo la presencia de paternariados público-privados establecidos como GAL, la efectiva gestión de los fondos para el desarrollo rural, la capacidad institucional y la buena gobernanza (Sánchez, Gallardo y Ceña, 2014). Todo ello en un contexto marcado tanto por las múltiples variaciones terminológicas usadas como por la desconexión existente 
entre los diferentes cuerpos teóricos que abordan este nuevo enfoque del desarrollo rural (Guinjoan, Badía, Tulla, 2016).

Por otra parte, y en líneas generales, se considera que la aplicación de esta filosofía y enfoque para el desarrollo rural ha sido muy exitosa. Desde sus comienzos ha resaltado como un instrumento ejemplarizante en la práctica del desarrollo local participativo y endógeno en el que las comunidades rurales se apoyan para determinar sus propios caminos de desarrollo a través de un uso racional y sostenible de los recursos locales. Como sugiere Ray (1998), LEADER tiene el potencial de democratizar el desarrollo rural. Otros autores también resaltan como mayores logros la descentralización, el empoderamiento de las comunidades rurales y la renovación de su capital social (Shucksmith, 2000; Ramos y Delgado, 2003; Farrell y Thirion, 2005; entre otros).

Ahora bien, la participación local ha sido más formal que real (Midmore, 1998), donde las tendencias, las decisiones y los controles descendentes —en gran parte, de la administración regional— han reducido la toma de decisiones local (Navarro, Woods y Cejudo, 2016), siendo esta Iniciativa controlada por las élites y lobbies políticos y económicos locales, convirtiéndose en un «instrumento político, económico y social» (Esparcia, Noguera y Pitarch, 2000: 96) que refuerza la estructuras de poder y deja al margen a los colectivos más desfavorecidos (Shucksmith, 2000; Dargan y Shucksmith, 2008; Shortall, 2008; y Furmankiewicz, 2010; entre otros). Por otra parte, los constreñimientos financieros y burocráticos también han limitado la capacidad para realizar cambios significativos (Ray, 1998; y Navarro, Woods y Cejudo, 2016).

Acercándonos a nuestra temática en cuestión, el estudio de los «otros proyectos», «los proyectos fallidos», desconocemos que existan estudios específicos al respecto; aunque no podemos obviar que, indirectamente e implícitamente, sí se ha abordado el fondo de la cuestión. Como se ha señalado anteriormente, algunos autores ya han subrayado que existe, por una parte, una "project class» (Dargan y Shucksmith, 2008: 285), una «clase de proyecto», incluyendo aquí principalmente los miembros de los GAL (equipo técnico, integrantes de la asociación, actores tanto públicos como privados), privilegiados del sector público y privado, con alta capacidad económica, de conocimientos e innovaciones, que controlan y están informados sobre las inversiones de LEADER, y que por otra, en el extremo opuesto, existen otra serie de colectivos, de grupos sociales, que quedan bastante al margen de ésta, que frecuentemente, y como se comprobará más adelante, van a ser mayoría en los proyectos diseñados y aprobados, pero finalmente no llevados a cabo. Así, por ejemplo, Viladomiu et al (2010: 281) señalan que «el establecimiento de primas para las mujeres ha tenido poca incidencia como determinante de una mayor participación de este colectivo». 
Vaillant y Lafuente (2007), por otra parte, apuntan, de forma genérica, que el fracaso empresarial, en definitiva, que la mayor o menor existencia de proyectos fallidos en ámbitos rurales, se debe más a pensar en los beneficios a corto plazo, en la no existencia de cultura de emprendimiento y de intolerancia al fracaso en estos territorios. En otro sentido, y pensando en el enfoque de género, Ariga, Lafuente y Vaillant (2009), señalan la menor tendencia de las mujeres a ser empresarias rurales, aunque, su menor miedo al fracaso empresarial.

\section{Fuentes, METOdOlOGía y ÁREA DE ESTUDio}

La gran extensión de Andalucía, $87.268 \mathrm{~km}^{2}$, y 50 GAL nos hizo que decidiéramos trabajar con dos escalas de análisis: a nivel regional y a nivel subregional para el caso de la provincia de Granada. La región está cubierta extensamente por el enfoque LEADER y sus respectivos GAL, lo que suministra una gran diversidad de casos y tipos de implementación de ésta, 770 municipios, en mayor medida acogidos a LEADER+ frente a un menor número para PRODER-A. Así, los territorios analizados poseen una posición periférica, no solo a nivel del conjunto de la UE, sino también a nivel nacional, con una gran lejanía respecto a los principales centros de decisión política y económica. Por otra parte, esta región es predominantemente rural en términos de extensión superficial, cubriendo gran cantidad de territorios de montaña y de altiplanicies (Cordilleras Béticas y Sierra Morena). Esto ha permitido una significativa dispersión de los patrones de asentamiento, con núcleos de población que generalmente no superan los 5.000 habitantes, aunque también nos encontramos con la presencia de agrociudades, muchas de ellas cabeceras comarcales, en la mayor parte de las ocasiones con más de 20.000 habitantes. Históricamente, el sector agrario ha sido la principal actividad económica de la región, permaneciendo actualmente su importancia, no tanto en términos económicos (PIB), y sí desde el punto de vista social, cultural y patrimonial. No conviene perder de vista que, directa e indirectamente, ligado al sector agrario, las explotaciones agrarias en general y las de pequeño y mediano tamaño, en particular, reciben una serie de ayudas y percepciones ligadas, por un lado, a la Política Agraria Comunitaria a través de su primer pilar, y por otro lado, al Subsidio de Desempleo Agrario para el caso de los trabajadores eventuales agrarios por cuenta ajena (Cejudo, Navarro, Maroto 2016; Cejudo, Navarro, Cañete, 2016). Aun así, en el medio rural andaluz, los ingresos per cápita y los estándares de vida son significativamente más bajos que la media nacional.

La agricultura en Andalucía gira en torno a productos típicamente mediterráneos (olivar, cereales y agricultura intensiva en la costa). El 
desempleo en las áreas rurales está por encima del 20\%, incluso antes de la coyuntura actual de crisis económica (Junta de Andalucía, 2008). La alta tasa de desempleo es una constante del devenir histórico de esta tierra y tiene un importante componente estructural, lo que se conoce como «cuestión agraria» en la literatura especializada. Ésta se sustenta históricamente, en una injusta distribución de la propiedad de la tierra que, si bien tradicionalmente ha sido utilizada por la historiografía como la «principal expresión» de la desigualdad social en el interior de la zonas rurales andaluzas, en los últimos años está siendo cada vez más discutida desde esta perspectiva (González de Molina, Infante y Herrera, 2014; Garrabou et al., 2014), por lo que se plantea la necesidad de abordarla desde nuevos planteamientos y dimensiones que incorporen a la tradicional equidad social, la defensa de la sostenibilidad a la hora de producir y alimentarnos (Arnalte, Baptista y Garrabou, 2012; González de Molina, 2014). Ello ha venido provocando en Andalucía que un gran número de personas queden atrapadas en el círculo vicioso del desempleo o subempleo estructural dentro del agro andaluz. Ello se traduce en que estos trabajadores estén empleados una parte del año y para el resto sean dependientes de ayudas y subsidios (Ramos y Romero, 1995). Aun así, el Plan de Empleo Rural, que incluye el subsidio de desempleo agrario, ha sido y es aquí vital para el mantenimiento de las poblaciones rurales.

Otro elemento importante a considerar es que la política de desarrollo rural está determinada más por el gobierno autonómico y regional, que por el gobierno nacional.

En cuanto a las fuentes de información empleadas han sido básicamente tres. En primer lugar, la relación de proyectos no ejecutados durante el periodo 20022008 para toda Andalucía por parte de sus 50 GAL, proporcionada por el Gobierno de esta región, en concreto, la Dirección General de Desarrollo Sostenible de la Consejería de Agricultura, Pesca y Desarrollo Rural de la Junta de Andalucía. Aunque el periodo oficial es el 2000-2006, en realidad la aplicación de los programas en nuestra área de estudio no se inició hasta 2002 y se prolongó hasta 2008. Nos circunscribimos a este periodo de tiempo por ser el más reciente del que se dispone de información actualizada, detallada y veraz de este tipo de proyectos. Del periodo posterior, 2008-2015, aún no existe tal documentación. En detalle, sería la Iniciativa LEADER + (Eje 1 y 2.1) y el Programa de Desarrollo Rural de Andalucía (PRODER-A), incluido dentro del Programa Operativo Integrado de Andalucía en su Eje 7: medida 7.55 sobre desarrollo endógeno de zonas rurales en el ámbito agrario: diversificación agraria, y la 7.59 sobre desarrollo endógeno de zonas rurales no ligado a actividades agrarias. Ello se ha concretado en una relación de 1.281 registros de proyectos fallidos —entendiendo por tales, como ya 
se dijo, los aprobados y con contrato de realización suscrito con el correspondiente GAL pero finalmente no ejecutados- sobre un total de 9.502 aprobados, el 13,5\%.

En segundo lugar, con el objeto de contrastar los datos estadísticos anteriores y concretar algunas de las informaciones, esta documentación se ha complementado con trabajo de campo, a través del estudio de los expedientes fallidos en los archivos de cada una de las sedes de seis GAL de la provincia de Granada (Alfanevada, Alpujarras, Guadix, Poniente, Valle de Lecrín-Temple y Vega-Sierra Elvira), exceptuando los Montes y Altiplano, que por motivos burocráticos y de gestión no permitieron consultarlos, probablemente también a causa de la no existencia de tales documentos en la actualidad. Es decir, un $12 \%$ del total de GAL de la región. Los GAL seleccionados reúnen también una amplia variedad de casuísticas: comarcas de montaña, altiplanicies, zonas de valle y transición, hoyas interiores, áreas periurbanas e incluso metropolitanas, y zonas de transición y traspaís de la costa.

Se revisaron in situ la documentación correspondiente a cada uno de estos proyectos. En definitiva, un total de 128 proyectos, el 10\% del total de los existentes para Andalucía. El trabajo de archivo fue arduo y dificultoso por la lejanía en el tiempo, casi 10 años, la necesidad de búsqueda por nuestra parte y los técnicos de cada GAL, dentro de unos archivos escasamente organizados, salvo raras excepciones.

$\mathrm{Y}$ en tercer lugar, se abordaron una serie de entrevistas en profundidad a técnicos y gerentes de cada uno de los GAL señalados, en total doce, dos por cada GAL, cuestionándoles sobre algunos aspectos que no quedaban claros en la documentación de cada uno de los expedientes, bien por estar incompletos bien por el carácter «general»y «genérico» de, en concreto, las causas últimas que motivaron su no ejecución, aspecto éste en que se hizo mayor hincapié en esta fase de la investigación. Técnicos y gerentes, con la documentación del expediente sobre la mesa, nos señalaron, para la inmensa mayoría de los casos, los motivos principales que abocaron a la caída de tales iniciativas. Así, se ha podido contrastar qué es lo que hay detrás de términos tan genéricos como «renuncia» a la hora de catalogar la no ejecución de estos proyectos.

«Protocolo» Y FASES De TRAMitación De Un PRoyecto LEADER/PRODER. ¿EN QUÉ ETAPA QUEDARON LOS EXPEDIENTES ESTUDIADOS?

Antes de iniciar el análisis de los expedientes consultados, es necesario conocer el proceso administrativo y las fases de tramitación que debieron seguir, y que superaron como proyectos LEADER y PRODER, además 
de visualizar el momento exacto en el que quedaron paralizados. Así, las solicitudes de subvenciones estudiadas, son ayudas directas, a fondo perdido, destinadas a cofinanciar inversiones que realicen un emprendedor ya sea persona física o jurídica o bien entidades públicas o privadas sin ánimo de lucro. En relación al proceso de tramitación, los proyectos estudiados han alcanzado la fase de formalización del contrato de ayuda entre el GAL y el beneficiario de la subvención, pero no han podido o querido cumplir alguna de las siguientes condiciones: ejecutar el proyecto de conformidad con las obligaciones pactadas en el contrato; iniciar la ejecución de la inversión en el plazo máximo de 3 meses y finalizarla en el plazo máximo de 18 meses a contar desde la firma del contrato; presentar los documentos justificativos de la realización total de la actuación subvencionada; mantener el destino de la inversión durante, al menos, 5 años; llevar un registro contable de las operaciones económicas relacionadas con la inversión subvencionada; y comunicar a la opinión pública que la inversión está subvencionada por la administración andaluza y por la UE.

Es decir, los proyectos habían presentado la solicitud de subvención, momento en el cual también presentaron la memoria descriptiva del proyecto, las facturas pro-forma, el proyecto de obra en su caso, la documentación relativa a la identidad de la persona solicitante, declaraciones responsables varias, el certificado de la entidad bancaria donde se realizará la transferencia y la acreditación de que se encuentra al corriente de sus obligaciones fiscales. Tras ello, se realiza un análisis inicial de la solicitud en un plazo de un mes para estimar si se adecúa o no a las actuaciones subvencionables. A continuación se procede al levantamiento del acta de no inicio de la inversión, mediante la cual se comprueba que la inversión objeto de subvención no se encuentra iniciada, ya que no se otorgará ésta si la intervención se encuentra iniciada. Posteriormente, se informa a la Delegación Provincial de Agricultura y Pesca, en nuestro caso, de la Junta de Andalucía, para que en un plazo de un mes comunique al GAL si la intervención cumple con las condiciones de legalidad. En el siguiente paso, el equipo técnico del GAL elabora un informe técnicoeconómico de la solicitud a través del cual se determina la subvención final que puede alcanzar la inversión. El Responsable Administrativo Financiero (RAF) del GAL realiza una reserva presupuestaria a favor del solicitante, siendo el Consejo del GAL el que acuerda la concesión de la subvención en base al Informe TécnicoEconómico y el Informe del RAF. El acuerdo del Consejo del GAL informa al solicitante de la aprobación de la subvención concedida. Y, por último, se alcanza la fase anteriormente señalada de formalización del contrato de ayuda, momento en el que se quedaron los proyectos estudiados. 
Es decir, estos expedientes no llegaron a alcanzar el resto de fases: solicitud de pago de la subvención, el acta de finalización de la inversión, y por último, la certificación de gastos. Por regla general, el proceso puede dilatarse hasta dos años, o incluso más, tal y como se aprecia del cronograma considerado de un proyecto fallido tipo (tabla 1), resultando finalmente el proyecto desestimado.

TABLA 1

PROTOCOLO DE TRAMITACIÓN SEGUIDO POR UN EXPEDIENTE FALLIDO TIPO

\begin{tabular}{|l|l|l|}
\hline Fecha & \multicolumn{1}{|c|}{ Actor } & \multicolumn{1}{c|}{ Procedimiento } \\
\hline $25 / 09 / 06$ & Promotor & Entrada del expediente \\
\hline $25 / 09 / 06$ & Gerente & Acta de no inicio \\
\hline $20 / 12 / 06$ & Gerente & Elaboración consulta de subvencionabilidad \\
\hline $26 / 01 / 07$ & Delegación Provincial & Redacción CAP (subsanación) \\
\hline $12 / 02 / 07$ & Gerente & Informe técnico-económico \\
\hline $13 / 02 / 07$ & Responsable Administrativo Financiero (RAF) & Fiscalización proyecto por del RAF del gasto \\
\hline $18 / 02 / 07$ & Junta Directiva & Acuerdo Junta Directiva aprobación ayuda \\
\hline $23 / 02 / 07$ & Gerente & Notificación aprobación de ayuda \\
\hline $06 / 03 / 07$ & Promotor & Aceptación de ayuda \\
\hline $06 / 03 / 07$ & Promotor & Firma contrato de ayuda \\
\hline $25 / 02 / 08$ & Gerente & Notificación de ausencia e irregularidades \\
& de justificación \\
\hline $11 / 09 / 08$ & Gerente & Requerimiento de la misma información \\
\hline $18 / 06 / 08$ & Promotor & Remite información parcial \\
\hline $10 / 07 / 08$ & GAL & Rescisión del contrato \\
\hline $15 / 07 / 08$ & Junta Directiva & Acuerdo de rescisión \\
\hline $21 / 07 / 08$ & Gerente & Notificación de acuerdo \\
\hline
\end{tabular}

Fuente: Grupo de Acción Local de las Alpujarras.

\section{Resultados}

\section{El análisis a escala regional}

A nivel regional, y como se ha señalado anteriormente, los 1.281 registros de proyectos fallidos, sobre un total de 9.502 inicialmente aprobados, se corresponden con un $13,5 \%$. En su mayor parte, los proyectos aprobados, pero no ejecutados con subvención certificada, correspondían a PRODER (el 75,7\% 
de los proyectos fallidos, 970) (tabla 2). La tasa de éxito fue ligeramente más alta en LEADER (88,4\%) frente a PRODER (85,8\%).

De las cuatro categorías «oficiales» de causas que recoge la catalogación de los proyectos fallidos por parte de la administración andaluza, la más frecuente es la de «Renuncia», ya que como tal se consideran el 90,6\% de los casos (1.161 de 1.281) (tabla 3). Sin embargo la realidad es más compleja, como se señalará más adelante. Aunque minoritarias las otras modalidades, «finalizado», «desestimado» o «sin finalizar», recogen situaciones administrativas variadas, desde aquellas en las que se va a proceder a tramitar el reintegro de las subvenciones concedidas por no justificar adecuadamente los pagos a sus proveedores, a aquellas en las que falta administrativamente alguna documentación para certificar totalmente su ejecución. Así, si mientras en los proyectos de LEADER casi todos los motivos se relacionan con «renuncia», en el caso de PRODER, en uno de cada diez, la causa de su no ejecución está relacionada con la «no finalización» de la inversión.

Atendiendo al tipo de emprendedor, se constata una cierta concentración de los proyectos fallidos en los grupos de autónomos $(35,4 \%)$, sociedades mercantiles $(17,4 \%)$ y corporaciones locales $(14,7 \%)$. Los datos de mayor interés, sin embargo, se obtienen de su comparación con los proyectos aprobados en los mismos grupos de destinatarios. Los resultados obtenidos corroboran algunas de las conclusiones obtenidas en trabajos anteriores relativas tanto a la menor implicación de los grupos sociales más necesitados (Navarro et al., 2016), como al control de la iniciativa por los lobbies económicos y políticos locales (Esparcia et al., 2000: 96); es decir, de la existencia de una «project class» (Dargan y Shucksmith, 2008: 285) frente a una «non project class» no tan presente en tales iniciativas. Así, los porcentajes de fallidos en relación con el total de aprobados son muy superiores para las autónomas mujeres jóvenes $(27,7 \%)$ y autónomos hombres jóvenes $(26,4 \%)$ respecto al total de autónomos hombres o de mujeres ( $21,5 \%$ en ambos casos), valor también ya elevado de por sí. También tenemos porcentajes de fracaso muy elevados para el caso de las sociedades mercantiles lideradas por mujeres jóvenes $(27,9 \%)$. De esta forma, el perfil de destinatario fallido se puede asimilar al de un empresario/emprendedor joven que trata de crear empresa, predominando las mujeres, y que adopta fórmulas jurídicas, básicamente, de autónomo o de comunidades de bienes. Ahora bien, el perfil de sexo y edad de los autónomos con proyectos caídos es muy similar al correspondiente al de este colectivo para las actuaciones finalmente ejecutadas $(40,2 \%$ de autónomos mujeres tanto en proyectos ejecutados con subvención como en fallidos) (tabla 4). 

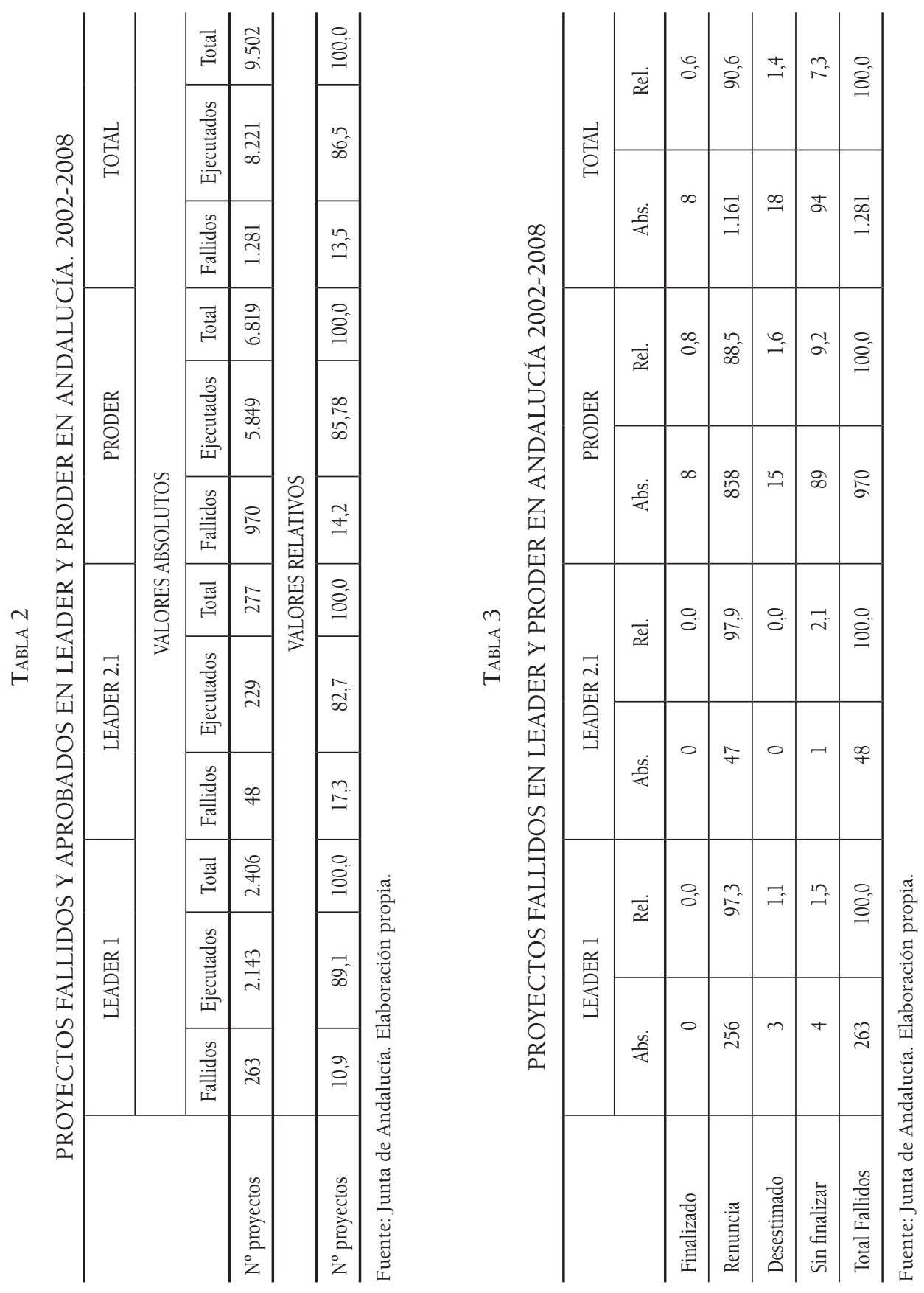

Estudios Geográficos, Vol. LXXIX, 284, pp. 141-166, enero-junio 2018 ISSN: 0014-1496, eISSN: 1988-8546, doi: https//doi.org/10.3989/estgeogr.201806 
TABLA 4

PROYECTOS FALLIDOS ATENDIENDO AL TIPO DE DESTINATARIO EN LEADER Y PRODER DE ANDALUCÍA. 2002-2008

\begin{tabular}{|c|c|c|c|c|c|c|}
\hline Tipo de destinatario & Fallidos & Ejecutados & Aprobados & $\mathrm{Fall} / \mathrm{Ejec}$ & Fall/Apro & \%_Fall \\
\hline 0.1 Autónomo mujer & 129 & 471 & 600 & 27,39 & 21,50 & 10,07 \\
\hline 0.2 Autónomo hombre & 192 & 701 & 893 & 27,39 & 21,50 & 14,99 \\
\hline 0.3 Corporación local & 188 & 2066 & 2254 & 9,10 & 8,34 & 14,68 \\
\hline $\begin{array}{l}0.4 \text { Cooperativa o Sociedad Agrario Transformación } \\
\text { y Soc. Responsabilidad Limitada }\end{array}$ & 26 & 211 & 237 & 12,32 & 10,97 & 2,03 \\
\hline 0.5 Sociedad Mercantil & 223 & 1107 & 1330 & 20,14 & 16,77 & 17,41 \\
\hline 0.6 Sociedad laboral & 37 & 176 & 213 & 21,02 & 17,37 & 2,89 \\
\hline 0.7 GDR o asociaciones & 126 & 2030 & 2156 & 6,21 & 5,84 & 9,84 \\
\hline 0.8 Otras entidades (C.B.) & 54 & 359 & 413 & 15,04 & 13,08 & 4,22 \\
\hline 1.0 Autónomo mujer joven & 52 & 136 & 188 & 38,24 & 27,66 & 4,06 \\
\hline 2.0 Autónomo hombre joven & 80 & 223 & 303 & 35,87 & 26,40 & 6,25 \\
\hline 4.0 Cooperativa o SAT (+25\% son jóvenes). & 5 & 23 & 28 & 21,74 & 17,86 & 0,39 \\
\hline 4.1 Cooperativa o SAT (+ $25 \%$ son mujeres) & 7 & 31 & 38 & 22,58 & 18,42 & 0,55 \\
\hline 4.2 Entidad de economía social & 2 & 15 & 17 & 13,33 & 11,76 & 0,16 \\
\hline 5.0 Sociedad Limitada & 20 & 99 & 119 & 20,20 & 16,81 & 1,56 \\
\hline 5.1 Sociedad mercantil (+25\% son jóvenes) & 57 & 267 & 324 & 21,35 & 17,59 & 4,45 \\
\hline 5.2 Sociedad mercantil (+25\% son mujeres jóvenes) & 19 & 49 & 68 & 38,78 & 27,94 & 1,48 \\
\hline 6.0 Otras Sociedades mercantiles & 10 & 40 & 50 & 25,00 & 20,00 & 0,78 \\
\hline 6.1 Sociedad laboral & 23 & 60 & 83 & 38,33 & 27,71 & 1,80 \\
\hline 6.2. C.B. & 9 & 14 & 23 & 64,29 & 39,13 & 0,70 \\
\hline 7.0 Asociación o fundación (+25\% son jóvenes) & 2 & 6 & 8 & 33,33 & 25,00 & 0,16 \\
\hline 7.1 Asociación o fundación (+25\% son mujeres) & 6 & 63 & 69 & 9,52 & 8,70 & 0,47 \\
\hline 7.2 Otras Asociaciones & 0 & 4 & 4 & 0,00 & 0,00 & 0,00 \\
\hline 8.0 Otras entidades ( $+25 \%$ son jóvenes) & 5 & 16 & 21 & 31,25 & 23,81 & 0,39 \\
\hline 8.1 Otras entidades ( $+25 \%$ son mujeres) & 5 & 39 & 44 & 12,82 & 11,36 & 0,39 \\
\hline 8.2 Otras & 4 & 15 & 19 & 26,67 & 21,05 & 0,31 \\
\hline TOTAL & 1.281 & 8.221 & 9.502 & 15,58 & 13,48 & 100,00 \\
\hline
\end{tabular}

Fuente: Junta de Andalucía. Elaboración propia. 


\section{El análisis a escala subregional o provincial}

Si descendemos ahora al análisis de los seis GAL de la provincia de Granada, y se detalla aún más el perfil de estos emprendedores fallidos, se puede afirmar, en primer lugar, que dentro de los productivos, 92 sobre un total de 128 abordados, el 60,3\% (44 de 73 conocidos) eran nuevos emprendedores, ya que la inversión se destinaba a empresas de nueva creación, cifra bastante superior a la que podemos encontrar para el caso de los proyectos finalmente ejecutados donde las nuevas empresas creadas apenas alcanzaron un 25\% (23,6\%). Ahora bien, ello no quiere decir que estos promotores no tuvieran experiencia profesional, al contrario, un $81,5 \%$ de ellos tenían ya una trayectoria laboral en el mismo sector mientras que solo un 4,6\% no tenía experiencia y era su primer trabajo y, finalmente, un $13,8 \%$ procedían de otro sector, aunque con relaciones y semejanzas con la actividad donde se realiza la inversión.

En lo que respecta a las inversiones que se plantean (tabla 5), hay que reseñar, en primer lugar, la reducida inversión planteada en los proyectos liderados por mujeres autónomas de no más de 30 años, 22.568 euros, y que contrastan con las de hombres jóvenes autónomos (103.976 euros). En el primer caso tenemos ejemplos de aperturas de librería-papelería, peluquería, cafetería y laboratorio agroalimentario, mientras que en el segundo caso, la creación de un alojamiento rural, de una quesería artesanal, ampliación de una panadería, o la creación de un centro de formación con acceso a internet. En el extremo opuesto, las inversiones medias propuestas mayores las encontramos en las sociedades mercantiles donde más del $25 \%$ de sus miembros son jóvenes, con 475.066 euros, muy superiores a las anteriormente señaladas de autónomas mujeres jóvenes (representando éstas únicamente el 4,8\% respecto de aquellas), presentándose aquí expedientes tales como la puesta en marcha de un hotel, de un restaurante, o la modernización de una pasteleríapanadería. Llama la atención la escasa subvención planteada para estos proyectos de mujeres jóvenes autónomas, de apenas un 24,4\%, igual en el caso de sociedades mercantiles con más del $25 \%$ de jóvenes donde apenas se alcanza el 13,2\% de subvención. Y al contrario, dentro de los emprendedores privados, los que presentan mayores subvenciones son las sociedades mercantiles $(+25 \%$ mujeres), $37,2 \%$, y las autónomas mujeres de 30 años y más $(37,0 \%)$, siendo en este caso para el colectivo de GAL o asociaciones para el que se plantea una mayor subvención $(80 \%)$. 
TABLA 5

INVERSIONES PROPUESTAS POR TIPO DE EMPRENDEDOR EN LOS GAL ANALIZADOS (2002-2008)

\begin{tabular}{l|c|c|c}
\hline \multicolumn{1}{c|}{ Tipos de emprendedores } & $\begin{array}{c}\text { Número de } \\
\text { proyectos }\end{array}$ & $\begin{array}{c}\text { Inversión media } \\
\text { propuesta por } \\
\text { proyecto (euros) }\end{array}$ & $\begin{array}{c}\text { Subvención media } \\
\text { propuesta por } \\
\text { proyecto (\%) }\end{array}$ \\
\hline Autónomas mujeres (30 años y más) & 17 & 103.135 & 37,0 \\
\hline Autónomas mujeres (por debajo de 30 años) & 5 & 22.568 & 24,4 \\
\hline Autónomos hombres (30 años y más) & 24 & 69.168 & 29,6 \\
\hline Autónomos hombres (por debajo de 30 años) & 7 & 103.976 & 29,6 \\
\hline Cooperativas & 2 & 60.283 & 26,2 \\
\hline Sociedades mercantiles & 19 & 341.079 & 27,6 \\
\hline Sociedades mercantiles (+25\% jóvenes) & 6 & 475.066 & 13,2 \\
\hline Sociedades mercantiles (+25\% mujeres) & 2 & 103.956 & 37,2 \\
\hline Ayuntamientos & 15 & 66.009 & 41,0 \\
\hline GAL o asociaciones & 15 & 37.748 & 79,8 \\
\hline Otros & 16 & 99.098 & 39,1 \\
\hline Total & 128 & 139.306 & 30,6 \\
\hline
\end{tabular}

Fuente: GAL de Alfanevada, Alpujarras, Guadix, Poniente y Vega-Sierra Elvira. Elaboración propia.

El análisis de sexo y edad en los proyectos fallidos consultados, con finalidad productiva y solicitados por autónomos (figura 1), muestra una abrumadora presencia de los presentados por hombres $(67,4 \%)$, frente a un reducido peso de los de mujeres $(32,6 \%)$, muy similar al que encontramos en los que sí se llevaron a cabo para Andalucía, 39,6\% para mujeres frente a un 60,4\% para hombres.

Para las mujeres autónomas, tenemos ejemplos de aperturas de nuevos negocios (69\% empresas de nueva creación), proponiendo la creación de cafeterías, hoteles y alojamientos rurales, comida a domicilio, talleres de cerámica artesanal y textil, comercio de ropa infantil, como ejemplos más significativos. En el caso de los liderados por jóvenes, hombres o mujeres, el intento de crear nuevas empresas predomina en estos proyectos (80\%).

Por edades, los que se encuentran por debajo de 30 años, son minoritarios, $19,6 \%$, predominando aquí los solicitados por hombres, bastante semejante al perfil de los finalmente llevados a cabo, 23,5\%, también abundando aquí los desarrollados por personas de sexo masculino. 
FIGURA 1

SEXO Y EDAD EN LOS PROYECTOS FALLIDOS PRODUCTIVOS DE AUTÓNOMOS EN LOS GAL DE ALFANEVADA, ALPUJARRAS, GUADIX, PONIENTE Y VEGA-SIERRA ELVIRA

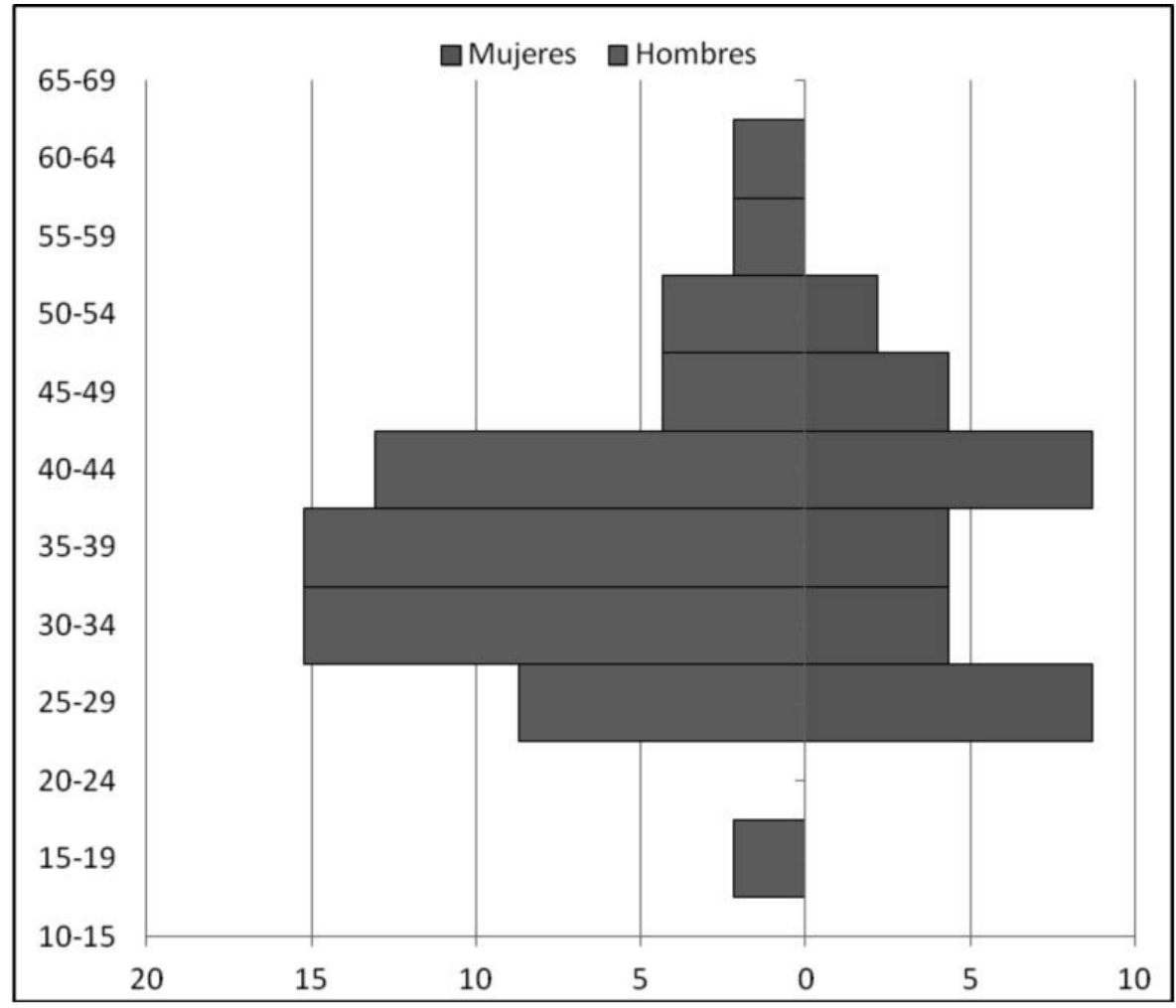

Fuente: GAL de Alfanevada, Alpujarras, Guadix, Poniente y Vega-Sierra Elvira. Elaboración propia.

Por otra parte, sobre el origen geográfico de los emprendedores, dentro de los que realizaron actuaciones productivas, la mayor parte procedían del propio territorio del GAL (85,3\%), principalmente del mismo municipio donde realizan la inversión $(74,7 \%)$, siendo los menos los que proceden de otro GAL $(5,3 \%)$, de otra provincia (5,3\%), u otro estado $(4,0 \%)$.

En lo que se refiere al nivel de estudios, conocido para un total de 48 de estos expedientes analizados en profundidad, un 39,6\% tenían estudios primarios, 22,9\% estudios secundarios de Formación Profesional, 12,5\% estudios secundarios de Bachillerato, y únicamente un $27,1 \%$ poseían estudios 
universitarios. Por tanto, y en líneas generales, estos emprendedores contaban con un nivel formativo bajo y medio-bajo.

En lo que respecta a las cuantías de las inversiones planteadas, en los GAL analizados aportan interesantes resultados (tabla 6). En primer lugar, la subvención media propuesta, es bastante superior en el caso de los proyectos fallidos, de un $33,2 \%$, frente a un $24,9 \%$ en los proyectos finalmente ejecutados. Por otra parte, la inversión media es bastante superior en los proyectos ejecutados, 205.137 euros frente a 137.840 euros en los fallidos, es decir un $33 \%$ inferior. Esta menor cuantía en los proyectos finalmente no desarrollados se aprecia también en la cuantía de los proyectos con mayor y menor inversión, siempre más reducida para el caso de los fallidos. En cuanto a la tipología de proyectos, coincide la finalidad de los que presentan mayor cuantía económica, destinados ambos a la construcción de un hotel.

TABLA 6

CUANTÍAS INVERSIÓN DESARROLLADAS Y PLANTEADAS EN PROYECTOS EJECUTADOS Y FALLIDOS PARA LOS GAL DE ALFANEVADA, ALPUJARRAS, GUADIX, PONIENTE Y VEGA-SIERRA ELVIRA (EUROS)

\begin{tabular}{l|c|c|c|c|c|c}
\hline $\begin{array}{c}\text { Tipos de } \\
\text { proyectos }\end{array}$ & $\begin{array}{c}\text { Inversión } \\
\text { media } \\
\text { desarrollada/ } \\
\text { planteada }\end{array}$ & $\begin{array}{c}\text { Inversión } \\
\text { mínima } \\
\text { desarrollada/ } \\
\text { planteada }\end{array}$ & $\begin{array}{c}\text { Inversión } \\
\text { privada media } \\
\text { desarrollada/ } \\
\text { planteada } \\
\text { (total) }\end{array}$ & $\begin{array}{c}\text { Inversión } \\
\text { privada media } \\
\text { desarrollada/ } \\
\text { planteada } \\
\text { (porcentaje) }\end{array}$ & $\begin{array}{c}\text { Caso de mayor } \\
\text { inversión } \\
\text { desarrollada/ } \\
\text { planteada } \\
\text { (concepto) }\end{array}$ & $\begin{array}{c}\text { Caso de mayor } \\
\text { inversión } \\
\text { desarrollada/ } \\
\text { planteada } \\
\text { (cuantía total) }\end{array}$ \\
\hline Ejecutados & $205.136,5$ & $1.329,4$ & $153.991,4$ & 75,1 & $\begin{array}{l}\text { Construcción } \\
\text { hotel }\end{array}$ & $1.452 .218,7$ \\
\hline Fallidos & $137.839,9$ & 755,4 & $92.095,6$ & 66,8 & $\begin{array}{l}\text { Rehabilitación } \\
\text { casa señorial } \\
\text { para hotel rural }\end{array}$ & $1.395 .100,0$ \\
\hline
\end{tabular}

Fuente: GAL de Alfanevada, Alpujarras, Guadix, Poniente y Vega-Sierra Elvira. Elaboración propia.

Por tipologías de proyectos, hay que señalar la mayor cuantía económica planteada para los de turismo y restauración (tabla 7), debido a que en este caso se plantean iniciativas tales como la rehabilitación de cortijos para hotel rural, o la construcción exnovo de hoteles o restaurantes, lo que exige un mayor esfuerzo inversor, con un promedio de propuesta inversora de 242.799 euros. Esa mayor apuesta económica no se acompaña de una subvención mayor, al poseer esta un volumen máximo absoluto, lo que da lugar a una 
menor subvención media propuesta (24,5\%). En el extremo opuesto, dentro de los productivos, los destinados a empresas de artesanía (cerámica y textil) apenas alcanzan una cuantía total de 15.511 euros, siendo la subvención ahora bastante superior $(39,0 \%)$. Les siguen, a continuación, en porcentaje de ayuda los de Valorización de la producción agraria (queserías, fábricas de embutidos, central hortofrutícola, mejora calidad en bodegas de vinos, etcétera), con un $37,2 \%$, y 88.833 euros de inversión media planteada. En PYMEs y servicios locales la casuística es muy amplia (Centro de Formación, Residencia Geriátrica, Televisión Comarcal, empresa de topografía, taller de carpintería metálica, de serigrafía, etc.), apareciendo una inversión promedio de 132.210 euros, y un 30,3\% de subvención media propuesta. Como es lógico, son los proyectos no productivos, destinados a Formación (en turismo rural, gestión de cooperativas agrarias, entre otros), los que presentan un mayor porcentaje de ayuda, $84,1 \%$, aunque una cuantía total muy reducida, 9.865 euros. Por último, en Patrimonio (inventario de patrimonio troglodítico; centro de interpretación del aceite y el patrimonio rural; sistema de información geográfica, identidad, señalización, estudios, libros, videos y páginas web interactivas sobre patrimonio, entre otros), la subvención planteada era también muy elevada $(77,5 \%)$, la cuantía total se encuentra en unos niveles bajos-medios (51.448 euros).

TABLA 7

INVERSIONES PROPUESTAS POR TIPO DE PROYECTO EN LOS GAL ANALIZADOS (2002-2008)

\begin{tabular}{|l|c|c|c|}
\hline \multicolumn{1}{|c|}{ Tipos de proyectos } & $\begin{array}{c}\text { Número de } \\
\text { proyectos }\end{array}$ & $\begin{array}{c}\text { Inversión media propuesta } \\
\text { por proyecto (euros) }\end{array}$ & $\begin{array}{c}\text { Subvención media } \\
\text { propuesta por proyecto (\%) }\end{array}$ \\
\hline Formación & 7 & 9.865 & 84,1 \\
\hline Integración social & 1 & 4.650 & 50,0 \\
\hline Patrimonio & 13 & 51.448 & 77,5 \\
\hline Turismo y restauración & 35 & 242.799 & 24,5 \\
\hline Artesanía & 2 & 15.511 & 39,0 \\
\hline PYMEs y servicios locales & 55 & 132.210 & 30,3 \\
\hline Valorización producción agraria & 15 & 88.833 & 37,2 \\
\hline Total & 128 & 139.306 & 30,7 \\
\hline
\end{tabular}

Fuente: GAL de Alfanevada, Alpujarras, Guadix, Poniente y Vega-Sierra Elvira. Elaboración propia. 
Sobre el grado de innovación hay que señalar que, atendiendo a lo comentado por los técnicos y gerentes de los GAL en el informe técnico-económico de la solicitud a través del cual se determina la subvención final que puede alcanzar la inversión, éste era medio-alto. Para la mitad de los proyectos fue de grado 2, medio, $(50,0 \%)$ o de grado 3 , alto $(43,1 \%)$, siendo minoritarios los que presentaban una innovación de grado 1 , baja (6,9\%). Y sobre el tipo de innovación predominante en estas actuaciones, se refiere, principalmente, en los expedientes las categorías de un «nuevo producto, servicio y valorización recurso ocioso», 75,9\%, y, a gran distancia, «maquinaria, herramientas o tecnología nueva», $22,2 \%$. Únicamente el $1,9 \%$ se relaciona con «mejores respuestas a problemas de la empresa».

El estudio en profundidad a través de las entrevistas a gerentes y técnicos. Motivos principales de desistimiento de los proyectos

Por último, de la entrevista en profundidad a gerentes y técnicos de estos seis GAL, a pesar de que la «renuncia» se convierte en el motivo «oficial» por el que se abandona el proyecto, o mejor dicho, la subvencionabilidad de éste por LEADER o PRODER, se puede afirmar que las razones últimas y principales que llevaron a incumplir el contrato son muy variadas, pudiéndose diferenciar hasta 13 tipos de casuísticas conocidas para los 92 proyectos productivos, del total de 128 (tabla 8). En primer lugar, casi la mitad de ellas - 40,2\% - estaban derivados de la inviabilidad económica/financiera y de la falta de planificación/ previsión por parte del promotor, directamente $14,1 \%$, o indirectamente, a través de la falta o irregularidad de documentación solicitada, un 26,1\% (de licencia de actividad, de apertura, proyecto viabilidad, alta en impuesto de actividades económicas, etc.). La irregularidad en la documentación también es reflejo de varios intentos de fraude o de cazar una subvención.

En segundo lugar, las relacionadas con la rigidez en tiempos y requisitos, agrupan a un $21,7 \%$, siendo la siguiente tipología de causas principales, situándose aquí: no cumple requisitos (4,3\%), plazos (15,2\%), e incumplimiento inicio de la inversión (2,2\%).

A continuación se presentan otra serie de múltiples razones $(21,8 \%)$ : la propia renuncia sin justificar motivo (8,7\%), acogerse a otra subvención (5,4\%), cambio de titular $(2,2 \%)$, causa mayor $(1,1 \%)$, desacuerdo entre emprendedores $(1,1 \%)$, realizarlo por otros medios sin subvención $(1,1 \%)$, la movilización social en contra $(1,1 \%)$ o el rechazar poner la placa indicativa de haberse acogido a la subvención a la entrada de su negocio $(1,1 \%)$. 
TABLA 8

MOTIVOS PRINCIPALES PARA LA NO REALIZACIÓN DE LOS PROYECTOS

PRODUCTIVOS FINALMENTE NO LLEVADOS A CABO, EN LOS GAL DE

ALFANEVADA, ALPUJARRA GRANADINA, GUADIX, PONIENTE, VALLE DE LECRÍN-TEMPLE Y VEGA-SIERRA ELVIRA EN LEADER Y PRODER EN ANDALUCÍA 2002-2008

\begin{tabular}{|c|c|c|c|}
\hline $\begin{array}{c}\text { Tipologías de causas } \\
\text { principales }\end{array}$ & Causas principales & $\begin{array}{l}\text { Números } \\
\text { absolutos }\end{array}$ & $\begin{array}{c}\text { Números } \\
\text { relativos (\%) }\end{array}$ \\
\hline \multirow[b]{2}{*}{$\begin{array}{l}\text { Inviabilidad económica/ } \\
\text { financiera y falta de } \\
\text { planificación }\end{array}$} & Inviabilidad financiera & 13 & 14,1 \\
\hline & $\begin{array}{l}\text { Falta o irregularidad documentación solicitada (proyecto } \\
\text { viabilidad, licencia apertura, licencia actividad, alta } \\
\text { en turismo, IAE, normas ISO, finalización proyecto, } \\
\text { justificación gastos, permisos...) }\end{array}$ & 24 & 26,1 \\
\hline \multirow{3}{*}{$\begin{array}{l}\text { Rigidez en tiempos y } \\
\text { requisitos }\end{array}$} & No cumple requisitos & 4 & 4,3 \\
\hline & Plazos & 14 & 15,2 \\
\hline & Incumplimiento inicio inversión & 2 & 2,2 \\
\hline \multirow{8}{*}{ Otras } & Renuncia & 8 & 8,7 \\
\hline & Acogerse a otra subvención & 5 & 5,4 \\
\hline & Cambio titular & 2 & 2,2 \\
\hline & Causa mayor & 1 & 1,1 \\
\hline & Desacuerdo entre emprendedores & 1 & 1,1 \\
\hline & Lo realiza por otros medios sin subvención & 1 & 1,1 \\
\hline & Movilización social en contra & 1 & 1,1 \\
\hline & Se niega a colocar la placa identificativa & 1 & 1,1 \\
\hline \multicolumn{2}{|l|}{ No se sabe } & 15 & 16,3 \\
\hline \multicolumn{2}{|c|}{ Total proyectos productivos } & 92 & 100,0 \\
\hline
\end{tabular}

Fuente: Entrevista en profundidad a gerentes y técnicos de los GAL de Alfanevada, Alpujarra Granadina, Guadix, Poniente, Valle de Lecrín-Temple y Vega-Sierra Elvira. Elaboración propia.

Los motivos de desistimiento en las mujeres autónomas se deben principalmente a la Inviabilidad económica/financiera y falta de planificación (65\%), y en segundo lugar a la Rigidez en tiempos y requisitos (20\%). Concretamente: falta o irregularidad en la documentación solicitada (50\%), Inviabilidad financiera (15\%) y Plazos (20\%). 
De estos proyectos, si se llevaron a la práctica por otros medios, ligeramente predominaron los que finalmente no se llegaron a ejecutar $(53,6 \%)$, siendo el resto ejecutados por otros medios, y dentro de estos últimos, un 10,7\% no funcionaron por lo que tuvieron que cerrar.

Es importante también considerar cada una de las casuísticas, reseñando ejemplos clave y tipo de proyectos que se han analizado. Así, en primer lugar, como se ha señalado, en materia de turismo, el proyecto fallido que preveía una mayor inversión era en el sector turístico, para la «Rehabilitación y ampliación de Casa Señorial para la puesta en marcha de un hotel rural», en el municipio de Atarfe, en el GAL de la Vega-Sierra Elvira. Con una inversión propuesta de 1.395.100 euros, y una subvención propuesta de 27.647 euros (apenas un 2,0\%). En éste, el motivo de renuncia se debió a la concesión de otra subvención por parte de la Consejería de Turismo, siendo incompatible con la ayuda LEADER. Otro caso digno de mencionar es el intento de «Creación de una empresa de reciclaje de residuos animales» en el municipio de Gor, proyecto ambiental, innovador y sostenible en muchos aspectos, y que finalmente no se llevó a cabo por la movilización social local en contra que finalmente presionó al Ayuntamiento para que denegara la licencia. Llama la atención también un emprendedor que desistió de la ayuda por no querer poner la Placa de haberse acogido a ayudas de PRODER, para la realización de un «taller y almacén eléctrico» en Beas de Granada, en el GAL de Alfanevada.

\section{DisCUSIÓN}

De lo abordado anteriormente podemos extraer una serie de reflexiones. En primer lugar, la alta tasa de éxito, ya que únicamente 1 de cada 10, —un 13,5\%, ligeramente más alta en LEADER que en PRODER - no se llevan finalmente a cabo con subvención de LEADER o PRODER una vez que los proyectos son aprobados y cuentan con contrato de realización con los GAL. Ahora bien, asumiendo como positivo lo anteriormente señalado, no conviene olvidar que se trata de proyectos que ya tienen un amplio recorrido administrativo, de control y gestión por parte de los GAL y que manifiestan y evidencian una clara intención inicial por parte de sus promotores de acogerse a este tipo de iniciativas. Entendemos que sería necesario un mayor seguimiento por parte de los técnicos de los GAL en la configuración y puesta en marcha de los proyectos que redujese aún más esta reducida tasa.

En segundo lugar, a pesar de que se parte de un nuevo objeto de estudio, los proyectos fallidos, se corroboran anteriores hipótesis. Así, frente a la presencia 
de una project class, de unas élites económicas locales que controlan la implementación de las iniciativas en cada uno de los territorios, aparece una non project classes, una serie de grupos y colectivos, nuevos emprendedores, a los que afectan en mayor medida los constreñimientos burocráticos y financieros impuestos para la aplicación de tales programas. Por tanto, en este aspecto, y a pesar de las contribuciones de los GAL en la mejora de la resiliencia de los territorios donde están asentados, su contribución es bastante más reducida, como lo manifiesta las dificultades que siguen existiendo a la hora de propiciar nuevas actividades y nuevos emprendedores lideradas por jóvenes y mujeres.

Por tanto, en tercer lugar, el perfil predominante en estos proyectos es el de jóvenes, mujeres y hombres, nuevos emprendedores autónomos que tratan de crear su nueva empresa, y por lo tanto, con un elevado desconocimiento de los procesos administrativos, aunque con trayectoria laboral en el sector o actividad, procedentes del propio territorio, con formación y cualificación media-baja. En definitiva, la mayor subvención no es lo importante para estos grupos, y más en proyectos donde las inversiones para crear sus futuras empresas son más reducidas que en promotores ya consolidados, sino otras cuestiones como la formación en emprendimiento y en cultura empresarial. Como señalan Cruces y Palenzuela (2006), concretamente para el caso de las mujeres emprendedoras, «se advierten entre ellas la tendencia al autoempleo, las ataduras a lealtades familiares y de grupo, una limitada formación empresarial y una escasa asunción del riesgo empresarial», además de producirse un «mantenimiento de los roles domésticos tradicionales» (Camarero y Sampedro, 2008: 102), como se aprecia en los proyectos potenciales a generar por ellas considerados en nuestro estudio: nuevas peluquerías, comidas a domicilio, artesanía textil y cerámica, elaboración de embutidos, restaurantes, hoteles y alojamientos rurales, entre otros.

Por otra parte, y en cuarto lugar, las causas principales para que no se hayan llevado a cabo estos proyectos son, en primer lugar, la inviabilidad económical financiera y la falta de planificación/previsión (40\%) y, en segundo lugar, las rigideces en tiempos y requisitos que establecen estas iniciativas (22\%). A este respecto es importante no olvidar que nos encontramos en un periodo de crecimiento económico y pre-crisis, sin apenas problemas de financiación por parte de las entidades bancarias, por lo que hemos de suponer que, cuando analicemos los datos del periodo 2007-2013, los proyectos fallidos serán mayores y se cebarán, aún más, con los colectivos ya reseñados que son los que cuentan con menor solvencia financiera en un contexto de restricción del crédito como el vivido durante este último periodo de programación. Por otra parte, señalar el alto porcentaje de proyectos que se ejecutaron por otros medios, 
el 46,3\%, lo que apunta, en primer lugar, a que la intención del emprendedor de acometer la actividad, independientemente de la percepción o no de la ayuda y su cuantía, y por tanto, y en segundo lugar, la reducida presencia, al menos en estos proyectos estudiados, de promotores caza subvenciones.

Ligado a lo anterior, en quinto lugar, los principios del desarrollo rural neoendógeno no se cumplen en la práctica de forma satisfactoria. Las rigideces en tiempos y documentación, causa principal de desistimiento en el $22 \%$ de los proyectos no llevados a cabo, la burocratización, los controles descendentes y las exigencias de la administración, principalmente regional, dificultan la adecuada implementación. Ello es así si tenemos en cuenta que, según señalan los entrevistados al repasar cada uno de los expedientes, un 36\% de ellos finalmente se llevaron a cabo bien recurriendo a otro tipo de ayudas ofertadas por otras instituciones bien renunciando a la subvención concedida por parte de LEADER y PRODER.

Además, se puede considerar que existió una relativa alta presencia en ellos de las especificidades de LEADER muy importantes relacionadas con la innovación, la puesta en valor de los recursos locales y la diversificación económica. Bien es cierto que se evidencia la reducida presencia de otras, también muy importantes y que podrían ayudar a explicar la caída de estos proyectos, como la integración y cooperación entre sectores y actores, el excesivo individualismo, la creación de cooperativas o la participación e iniciativa en común. De nuevo, se puede afirmar que los colectivos mayoritariamente representados en estos proyectos fallidos han tenido una participación más formal que real, en el programa, en la toma de decisiones, en la asociación del GAL, etc.

Y en lo que se refiere a la necesidad de mejorar la implementación de la filosofía LEADER y con ello de corregir sus errores, sería adecuado generar un apartado en los listados finales y evaluativos de estos programas en el que se describan las causas principales y últimas de su no puesta en marcha con financiación LEADER. Las denominaciones genéricas que se han venido utilizando reflejan, a nuestro entender, más un interés por «cumplimentar»el apartado correspondiente que el interés en generar y disponer de información valiosísima con la que poder corregir y mejorar la gestión de dichas iniciativas.

\section{Conclusiones}

En definitiva, se hace necesario reforzar la puesta en práctica del desarrollo rural neoendógeno, realzar sus fortalezas y corregir sus debilidades (control descendente, primacía élites económicas locales, reducida participación real, 
elevado individualismo proyectos, etc.), teniendo presentes, como pilares fundamentales, las especificidades de LEADER. Por otra parte, para fomentar los proyectos encabezados por jóvenes y mujeres, es más efectivo realizar actuaciones dirigidas a la formación empresarial y al fomento de la cultura emprendedora, al igual que una mayor priorización a la creación de nuevas actividades, de nuevas empresas.

Por otra parte, es imprescindible realizar un mayor seguimiento y planificación de estas actuaciones, flexibilizar los procesos administrativos relativos a los trámites documentales, así como propiciar un incremento de los tiempos para la ejecución de los proyectos en pro de mejorar la implementación de estas iniciativas y programas de desarrollo rural.

Y porúltimo, en futuros estudios sobre tal temática, sería adecuado profundizar en mayor medida en las casuísticas que rodean al desistimiento y caída en estos proyectos, realizando entrevistas en profundidad a los emprendedores, además de realizar el trabajo abarcando el periodo recién culminado, el 2009-2015, e incluyendo en el estudio a todas las propuestas que se presentan a los GAL para el inicio de su tramitación desde el momento inicial de su puesta en marcha, valorando, en la medida que la documentación nos lo permita, los momentos en los que estas propuestas van "cayéndose» en su tiempo de tramitación, así como las causas que concurrieron para que ello se produjese.

\section{Agradecimientos}

Este trabajo se ha realizado en el marco del proyecto de investigación «Programas de desarrollo y cambio rural en la Unión Europea: gobernanza, resultados y lecciones a compartir 2007-13» financiado por el Ministerio de Economía y Competitividad español dentro de su Programa de Excelencia, CSO2014-56223-P.

Agradecer también la disposición de los equipos técnicos y gerencia de los Grupos de Acción Local de Alfanevada, Alpujarras, Guadix, Poniente Granadino, Valle de Lecrín-Temple y Vega-Sierra Elvira.

\section{BiBLIOGRAFÍA}

Ariga, O., Lafuente, E. y Vaillant, Y. (2009): "Reasons for the relatively lower entrepreneurial activity levels of rural women in Spain", Sociologia Ruralis, 49(1), pp. 70-96. 
Arnalte, E., Baptista, F.O. y Garrabou, R. (2012): "Viejas y nuevas dimensiones de la cuestión de la tierra", Revista Española de Estudios Agrosociales y Pesqueros, 231, pp. 11-28.

Barke, M. y Newton, M. (1997): "The EU LEADER initiative and endogenous rural development: the application of the programme in two rural areas of Andalusia, Southern Spain", Journal of Rural Studies, 13, pp. 319-341.

Camarero, L. y Sampedro, R. (2008): “¿Por qué se van las mujeres? El continuum de movilidad como hipótesis explicativa de la masculinización rural”, Revista Española de Investigaciones Sociológicas, 124, pp. 73-105.

Cejudo, E. Navarro, F. y Maroto, J.C. (2016): "Perceptores del Subsidio de Desempleo Agrario y de la Renta Agraria en el sur de España: evolución, diferencias territoriales y estructura por edad y sexo", Ager, 20, pp. 33-72.

Cejudo, E. Navarro, F. y Cañete, J.A. (2016). "Distribución territorial de los trabajadores eventuales agrarios subsidiados en Andalucía: implicaciones geopoblacionales", Boletín de la Asociación de Geógrafos Españoles, 72, pp. 89-108.

Cheshire, L., Esparcia, J. y Shucksmith, M. (2015): "Community resilience, social capital and territorial governance", Ager, 18, pp. 7-38.

Cruces, C. y Palenzuela, P. (2006): "Emprendedoras rurales en Andalucía: posibilidades y límites de sus estrategias", Revista de Estudios Agrosociales y Pesqueros, 211, 239-305.

Dargan, L. y Shucksmith, M. (2008): "LEADER and innovation", Sociologia Ruralis, 48(3), pp. 274-291.

Esparcia, J., Noguera, J., y Pitarch, M. (2000): "LEADER en España: desarrollo rural, poder, legitimación, aprendizaje y nuevas estructuras", Documentos de Análisis Geográfico, 37, pp. 95-113.

European Economic Community (1988): "The future of rural society", Bulletin of the European Communities. COM (88) 501, pp. 1-68.

Farrell, G. y Thirion, S. (2005): "Social capital and rural development: from win-lose to win-win with the LEADER Initiative", en D. Schmied (ed.), Winning and losing. The changing geography of Europe's rural áreas, London, Ashgate, pp. 45-61.

Furmankiewicz, M., Thompson, N., y Zielinska, M. (2010): "Area-based partnerships in rural Poland: the post-accession experience", Journal of Rural Studies, 26, pp. 52-62.

Garrabou, R., Planas, J., Saguer, E. y Vicedo, E. (2014): "Propiedad de la tierra y desigualdad social en el mundo rural catalán de mediados de siglo XIX", Historia Agraria, 63, pp. 115-150.

González de Molina, M. (2014):"Introducción”, en M. González de Molina (coord.) La cuestión agraria en la historia de Andalucía, Sevilla, Centro de Estudios Andaluces, pp. 9-20.

González de Molina, M., Infante, J. y Herrera, A. (2014): "Cuestionando los relatos tradicionales: desigualdad, cambio liberal y crecimiento agrario en el Sur peninsular (1752-1901)", Historia Agraria, 63, pp. 55-88.

Goodwin, M. (1998): "The governance of rural areas: some emerging rural research issues and agendas", Journal of Rural Studies, 14, pp. 5-12. 
Guinjoan, E., Badía, A. y Tulla, A.F. (2016): "El Nuevo paradigma del desarrollo rural. Reflexión teórica y reconceptualización a partir de Rural Web”, Boletín de la Asociación de Geógrafos Españoles, 71, pp. 179-204.

High, C. y Nemes, G. (2007). "Social learning in LEADER: exogenous, endogenous and hybrid evaluation in rural development", Sociologia Ruralis, 47(2), pp. 103-119.

Junta de Andalucía (2008): Programa de Desarrollo Rural de Andalucía 2007-2013. Sevilla. Comité de Desarrollo Rural, Junta de Andalucía, 475 pp.

Marsden, T. (2009): "Mobilities, vulnerabilities and sustainabilities", Sociologia Ruralis, 49(2), pp. 113-131.

Marsden, T. y Murdoch, J.M. (1998): "The shifting nature of rural of rural governance and community participation", Journal of Rural Studies, 14, pp. 1-4.

Martínez, F., Sacristán, H. y Yagüe, J.L. (2015): “Are local action groups, under LEADER approach, a good way to support resilience in rural areas?", Ager, 18, pp. 39-63.

Midmore, P. (1998): "Rural policy reform and local development programmes: appropriate evaluation procedures", Journal of Agricultural Economics, 49(3), pp. 409-426.

Navarro, F., Woods, M. y Cejudo, E. (2016): "The LEADER Initiative has been a victim of its own success. The decline of the bottom-up approach in Rural Development Programmes. The cases of Wales and Andalusia", Sociologia Ruralis, 56(2), pp. 270-288.

Ramos, E. y Delgado, M. (2003): "European rural development. Programmes as a mean of strengthening democracy in rural areas. Walking towards justice: democratization in rural life", Research in Rural Sociology and Development, 9, pp. 135-157.

Ramos E. y Romero J. (1995): "Del "productivismo" al "ruralismo": una reflexión sobre la política agraria en Andalucía”, Revista de Estudios Agrosociales 169, pp. 175-212.

Ray, C. (1998): "New places and space for rural development in the European Union: an analysis of the UK LEADER II Programme", Working Paper, 34, pp. 1-51.

Ray, C. (2000): "The EU LEADER Programme: Rural Development Laboratory", Sociologia Ruralis, 40(2), pp. 163-171.

Ray, C. (2006): "Neoendogenous rural development in the EU", en P. Cloke, T. Marsden, y P. H. Mooney (eds.), Handbook of rural studies, London, Sage, pp. 295-336.

Sánchez, P., Gallardo, R. y Ceña, F. (2014): "Rural areas face the economic crisis: analysing the determinants of successful territorial dynamics", Journal of Rural Studies, 35, pp. 11-25.

Shortall, S. (2008): "Are rural development programmes socially inclusive? Social inclusion, civic engagement, participation, and social capital: exploring the differences", Journal of Rural Studies, 24 (4), pp. 450-457.

Shucksmith, M. (2000): "Endogenous development, social capital and social inclusion: perspectives from LEADER in the UK", Sociologia Ruralis, 40(2), pp. 208-218.

Vaillant, Y. y Lafuente, E. (2007): "Do different institutional frameworks contition the influence of local fear of failure and entrepreneurial examples over entrepreneurial activity?", Entrepreneurship and Regional Development, 19(4), pp. 313-337. 
Viladomiu, L., Rosell, J. y Francés, C. (2010): "Factores determinantes de la participación de la mujer como promotoras de proyectos LEADER +", Revista Española de Estudios Agrosociales y Pesqueros, 226, pp. 177-206.

Fecha de recepción: 18 de abril de 2017.

Fecha de aceptación: 5 de marzo de 2018. 